Universidade Federal Rural de Pernambuco (UFRPE), Rua Dom Manuel de Medeiros, s/n - Dois Irmãos, Recife - PE, Brasil

2 Bolsita PIBIC - FACEPE

${ }^{3}$ Universidade Federal de Pernambuco (UFPE), Av. Prof. Moraes Rego, 1235

Cidade Universităria, Recife - PE, Brasil

${ }^{4}$ Universidade de Pernambuco - UPE,

Av Gov. Agamenon Magalhães - Santo

Amaro, Recife,PE, Brasil

${ }^{5}$ Instituto Agronômico de Pernambuco

- IPA Av Gen San Martin, 1371

Bongi, Recife - PE, Brasil

* autor correspondente:

$\square$ rewysson.alves@gmail.com

\title{
Controle alternativo de Fusarium oxysporum com a utilização de extratos vegetais
}

\author{
Alternative control of Fusarium oxysporum with plant extracts
}

Rewysson Alves Ribeiro da Silva ${ }^{1,2^{*}}$, Mayara Goes Kettner ${ }^{3}$, Maria Luiza de Souza Lima4,

Luciana Gonçalves de Oliveira ${ }^{5}$, Emmanuelle Rodrigues Araújo ${ }^{5}$, Antonio Félix da Costa

RESUMO: O feijão-comum e o feijão-caupi são leguminosas de primordial importância na alimentação da população devido às suas fontes nutricionais e, em consequência, a geração de renda para pequenos produtores familiares. No entanto, as doenças são um dos fatores que podem afetar a produtividade do feijoeiro, como, por exemplo, a fusariose, que tem a aplicação de produtos químicos como método de controle mais utilizado. Métodos mais eficientes e que ocasionem menos danos à saúde humana e que degradem menos o ambiente são necessários. O objetivo deste trabalho foi avaliar o efeito fungitóxico de extratos de agave (Agave sisalana) e mastruz (Chenopodium ambrosioides) sobre o crescimento micelial e a esporulação de Fusarium oxysporum f. sp. phaseoli e Fusarium oxysporum f. sp. tracheiphilum. Para tanto, extratos hidroalcoólicos e aquosos foram adicionados ao meio de cultura BDA e em seguida utilizados em diferentes concentrações $(0 \%, 5 \%$, $10 \%$ e $20 \%$ ). As avaliações mostraram que os extratos à base de agave, tanto aquoso quanto hidroalcoólico, causam redução apenas em $F$. oxysporum f. sp. tracheiphilum, enquanto os extratos de mastruz (aquoso e hidroalcoólico) interferem significativamente no crescimento micelial e na esporulação dos três isolados, podendo ser utilizados no controle alternativo da fusariose.

PALAVRAS-CHAVE: Agave sisalana, Chenopodium ambrosioides, murcha-de-fusário.
ABSTRACT: Common beans and cowpea are important legumes in the population's diet due to their nutritional sources and income generation for small family farmers. Diseases such as Fusarium wilt are among of the factors that can affect the productivity of beans, and the application of chemicals is the most common control method. More efficient methods that cause less damage to human health and that degrade the environment less are necessary. This work aims to evaluate the fungitoxic effect of extracts of sisal (Agave sisalana) and "mastruz" (Chenopodium ambrosioides) on the mycelial growth and sporulation of Fusarium oxysporum f. sp. phaseoli and Fusarium oxysporum $f$. sp. tracheiphilum. For this, hydroalcoholic and aqueous extracts were used in different concentrations (0, 5, 10 and 20\%) and then added to the Potato dextrose agar. Evaluations showed that the aqueous and hydroalcoolic sisal is efficient for F. oxysporum f. sp. tracheiphilum; while the extracts of "mastruz" (aqueous and hydroalcoholic), showed that they significantly interfere in the mycelial growth and sporulation of the three isolates, being able to be used in the alternative control of fusarium wilt.

KEYWORDS: Agave sisalana, Chenopodium ambrosioides, Fusarium wilt.

\section{Introdução}

O feijão-comum (Phaseolus vulgaris L.) e o feijão-caupi (Vigna unguiculata (L.) Walp.) são espécies que apresentam a capacidade de adaptar-se às mais variadas condições ambientais, o que possibilita sua exploração sob diferentes sistemas de produção (FROTA et al., 2008; BRITO et al., 2009). Essas leguminosas têm papel importante na alimentação da população 
devido a seus princípios nutricionais e na geração de renda para pequenos produtores familiares (COMPAINHA NACIONAL DE ABASTECIMENTO, 2014). Os maiores produtores mundiais de feijão são Myanmar, Índia, Brasil, Estados Unidos, México e Tanzânia, responsáveis por $57 \%$ do total produzido no mundo (FOOD AND AGRICULTURE ORGANIZATION OF THE UNITED NATIONS, 2018). Contudo, sabe-se que as culturas de feijão-comum e do feijão-caupi são consideradas sensíveis tanto quanto ao déficit quanto ao excesso de água no solo, e sua produtividade é em função de diversos fatores, destacando-se as inter-relações entre solo-água-planta-atmosfera e manejo de irrigação, visando à máxima produção e à boa qualidade do produto (VALADÃO; KLAR, 1996).

Além dos fatores citados, as doenças se destacam na diminuição da produtividade do feijoeiro, especialmente as ocasionadas por fungos de solo, que podem causar prejuízos severos, como diminuição do estande, da qualidade fisiológica, nutricional e sanitária do produto colhido, afetando a produtividade, o preço e, consequentemente, a sua comercialização (Biazon, 2003). Os agentes causais dessas doenças apresentam estruturas de resistência que os ajudam a sobreviver no solo por vários anos, acarretando injúrias ao sistema radicular ou até mesmo atingindo a parte aérea das plantas (SARTORATO, 2007).

As doenças causadas pelo gênero Fusarium apresentam difícil controle devido à alta variabilidade genética do patógeno e sua ampla gama de hospedeiros como feijão, algodão, milho, soja, banana e outros, sendo necessária a combinação de várias medidas a fim de fornecer resultados efetivos (WENDLAND JUNIOR; LOBO JUNIOR; FARIAS, 2018).

$\mathrm{O}$ método mais utilizado para controle de doenças no campo ainda é o controle químico, porém o uso crescente e indiscriminado desses produtos tem oferecido riscos ambientais e à saúde humana e animal, por isso novas tecnologias que respeitem o ecossistema e não ofereçam risco de contaminação estão se tornando cada vez mais importantes para o controle de doenças e pragas como, por exemplo, o controle alternativo que utiliza extratos vegetais e óleos essenciais que apresentam baixa toxidez (GHINI; KIMATI, 2000; BERNARDO et al., 2002). Dentre essas medidas destacam-se o controle biológico e o controle alternativo que apresentam a vantagem de se utilizar agentes biológicos no controle de doenças em plantas (CAVERO et al., 2015; MILANESI et al., 2009).

Trabalhos desenvolvidos com extratos obtidos a partir de plantas são indicados no controle de fitopatógenos, tanto por sua ação fungitóxica direta, inibindo o crescimento micelial e a germinação de esporos (SCHWAN-ESTRADA; STANGARLIN, 2005), quanto pela indução de mecanismos de defesa da planta, fazendo com que ela apresente autodefesa (ROMEIRO, 2008). A eficácia de extratos aquosos de cravo-da-índia, alho e canela foi observada sobre os fitopatógenos Aspergillus sp., Penicillium sp., Colletotrichum sp., Phomopsis sp., Fusarium solani e Cercospora kikuchii (VENTUROSO et al., 2011). Por outro lado, Carnelossi et al. (2009) constataram o potencial dos óleos essenciais de Cymbopogon citratus, Eucalyptus citriodora, Mentha arvensis e Artemisia dracunculus no controle de Colletotrichum gloeosporioides em frutos de mamão, enquanto Silva e Bastos (2007) observaram atividade antifúngica de óleos essenciais de espécies de Piper sobre Moniliophthora perniciosa, Phytophthora palmivora e Phytophthora capsici.

Agave sisalana Perrine ex Engelm (Asparagales: Agavaceae) é uma planta herbácea, nativa da América Central e do México e encontrada em muitos países tropicais, como a Tanzânia e o Brasil (CHAND; TIWARY; ROHATGI, 1988). Resíduos líquidos e metabólitos secundários de sisal (alcaloides, saponinas e taninos) têm ação inseticida e antifúngica (ANDRESEN et al., 2015; LOPES et al., 2018).

A espécie medicinal Chenopodium ambrosioides L., também conhecida como mastruz, tem sido estudada a partir da sua caracterização fitoquímica para uso medicinal e no controle de pragas e doenças de plantas (MARINS et al., 2011), devido à presença de compostos químicos como flavonoides, alcaloides, saponinas e terpenos.

Diante disso, o objetivo deste trabalho foi avaliar o efeito da inibição do crescimento micelial e esporulação de Fusarium oxysporum f. sp. phaseoli e F. oxysporum f. sp. tracheiphilum por extratos de agave (Agave sisalana) e mastruz (Chenopodium ambrosioides) in vitro.

\section{Material e Métodos}

\section{Obtenção dos extratos}

o preparo dos extratos de mastruz foi conduzido após a secagem a $60^{\circ} \mathrm{C}$ em estufa por $48 \mathrm{~h}$. O material vegetal seco foi moído e armazenado para a elaboração dos extratos aquosos e hidroalcoólico, segundo a metodologia proposta por Celoto et al. (2008). Para o extrato com agave, foi utilizada a planta fresca pelo alto teor de fibras longas, assim suas folhas foram cortadas em pequenos pedaços, triturados em liquidificador e preparados os extratos aquosos e hidroalcoólico (LOPES et al., 2018).

Para obtenção do extrato hidroalcoólico, foi feita uma solução estoque de $30 \%$ com o seguinte procedimento: $150 \mathrm{~g}$ do material vegetal foram mantidos em repouso em $500 \mathrm{ml}$ de etanol a 70\% durante duas horas, quando a solução foi filtrada e levada ao banho-maria $\left(45^{\circ} \mathrm{C}\right)$ por $16 \mathrm{~h}$ para eliminação completa do álcool. Ao final, o volume inicial foi restabelecido adicionando-se água destilada e a solução foi esterilizada em autoclave a vapor fluente por 15 minutos. Posteriormente, o extrato hidroalcoólico foi adicionado ao meio batata-dextrose-ágar (BDA), ainda fundente, nas concentrações de 5\%,10\% e 20\% (CELOTO et al., 2008).

Para os extratos aquosos de agave e de mastruz, também foram preparadas soluções estoques a 30\% do material vegetal, com o seguinte procedimento: $150 \mathrm{~g}$ do material vegetal foram deixados em repouso em água destilada a $100{ }^{\circ} \mathrm{C}$ por $2 \mathrm{~h} \mathrm{e}$, posteriormente, filtrados (LOPES et al., 2018; CELOTO et al., 2008). Os extratos foram autoclavados utilizando a metodologia de vapor fluente por 15 minutos (VENTUROSO et al., 2011). Obtenção dos isolados fúngicos: foram utilizados dois isolados de Fusarium oxysporum f. sp. phaseoli isolados de plantas doentes do interior de Pernambuco, no município de São João, e F. oxysporum f. sp. Tracheiphilum foi isolado de planta doente do município de Goiana, PE. 
Análise da inibição por extratos vegetais sobre o crescimento micelial e esporulação de fitopatógenos in vitro

Ao meio de cultura BDA autoclavado, ainda fundente, foram adicionados extratos de agave e mastruz em diferentes concentrações $(5 \%, 10 \%$ e $20 \%)$ e distribuídos em placas de Petri. Para a testemunha, foi utilizado apenas o meio BDA distribuídos em placas de Petri.

Posteriormente, discos de $5 \mathrm{~mm}$ de diâmetro, contendo micélio dos fitopatógenos, foram transferidos para os centros das placas de Petri e incubados em câmara tipo BOD a $25^{\circ} \mathrm{C}$ por sete dias, na ausência de luz. Delineamento experimental: o delineamento experimental empregado foi inteiramente casualizado, sendo avaliados para cada planta/solvente quatro tratamentos (concentrações dos extratos: 0\%, 5\%, 10\% e 20\%) com cinco repetições (placas de Petri).

A avaliação constou da medição do crescimento radial das colônias em dois eixos ortogonais, posteriormente calculada a média e em seguida foram calculadas as percentagens de inibição do crescimento micelial (DOMINGUES et al., 2009; Edginton et al., 1971).

Para o bioensaio com esporulação dos fungos, os esporos foram quantificados utilizando-se câmara de Neubauer após sete dias de crescimento dos fungos nos respectivos meios e suas mencionadas concentrações.

Os dados foram analisados pelo teste de Tukey a 5\% de probabilidade utilizando-se programa Sisvar 5.6. (FERREIRA, 2019).

\section{Resultados e Discussão}

$\mathrm{O}$ crescimento micelial de $F$. oxysporum f. sp. phaseoli $1 \mathrm{e}$ F. oxysporum. f. sp. tracheiphilum em meio BDA com extrato aquoso de agave não apresentou diferença significativa ao nível de $5 \%$ de probabilidade, de acordo com o teste de Tukey, para as quatro concentrações $(0 \%, 5 \%, 10 \%$ e $20 \%)$. Quando se observou o crescimento micelial de $F$. oxysporum. f. sp. phaseoli 2 , verificou-se que o diâmetro da placa representando o crescimento do micélio foi maior com a adição do extrato de agave (Tabela 1). Resultados diferentes foram observados por Morais et al. (2010), constatando que o extrato aquoso de agave reduziu o crescimento micelial de $F$. oxysporum isolado de feijão-vagem à medida que a porcentagem de extrato aumentava no meio de cultura $(0 \%, 10 \%, 20 \%, 30 \%$ e $40 \%)$. Quanto ao crescimento micelial dos fungos testados com extrato hidroalcoólico do agave, houve redução significativa apenas para o F. oxysporum f. sp. tracheiphilum na concentração de $10 \%$ do extrato, de acordo com o teste de Tukey a 5\% (Tabela 1).

Os extratos aquosos e hidroalcoólico de matruz apresentaram resultados significativos contra os três isolados testados, Fusarium oxysporum f. sp. phaseoli (1 e 2) e Fusarium oxysporum f. sp. tracheiphilum, quanto ao crescimento micelial, de acordo com o teste de Tukey a $5 \%$ de probabilidade (Tabela 2 ). O extrato aquoso de mastruz reduziu significativamente o crescimento micelial para os três isolados à medida que as concentrações aumentaram, como observado na Tabela 2. Todos os isolados sofreram redução no diâmetro das colônias à medida que as concentrações do extrato hidroalcoólico de mastruz aumentaram

Tabela 1. Ação fungitóxica de extratos de agave sobre o crescimento micelial de Fusarium oxysporum.

\begin{tabular}{|c|c|c|c|c|c|c|c|c|c|c|}
\hline \multirow{3}{*}{ Espécies } & \multicolumn{5}{|c|}{ Extrato aquoso } & \multicolumn{5}{|c|}{ Extrato hidroalcoólico } \\
\hline & \multicolumn{5}{|c|}{ Concentrações (\%) } & \multicolumn{5}{|c|}{ Concentrações (\%) } \\
\hline & $\mathbf{0}$ & 5 & 10 & 20 & $\mathrm{CV} * \%$ & $\mathbf{0}$ & 5 & 10 & 20 & $\mathrm{CV} * \%$ \\
\hline $\begin{array}{l}\text { Fusarium oxysporum } \\
\text { f. sp. phaseoli } 1\end{array}$ & $8,85 a$ & $8,45 a$ & $8,85 a$ & $8,90 a$ & 3,80 & $9,00 \mathrm{a}$ & $9,00 a$ & $8,75 a$ & $8,90 a$ & 2,35 \\
\hline $\begin{array}{l}\text { F.oxysporum f. sp. } \\
\text { phaseoli } 2\end{array}$ & $6,85 b$ & $8,60 a$ & $9,00 a$ & $9,00 a$ & 5,81 & $9,00 a$ & $8,40 a$ & $7,95 \mathbf{a}$ & $7,90 a$ & 12,79 \\
\hline $\begin{array}{l}\text { F.oxysporum f. sp. } \\
\text { tracheiphilum }\end{array}$ & $8,30 a$ & $8,70 \mathbf{a}$ & $8,55 a$ & $8,80 a$ & 6,14 & $8,95 a$ & $8,90 a b$ & $8,30 b$ & 8,85ab & 4,29 \\
\hline
\end{tabular}

Na linha, mesma letra não difere estatisticamente entre si pelo teste Tukey $5 \%$ de probabilidade. $\mathrm{CV}^{*}$ : Coeficiente de Variação

Tabela 2, Ação fungitóxica de extrato de mastruz sobre o crescimento micelial de Fusarium oxysporum.

\begin{tabular}{|c|c|c|c|c|c|c|c|c|c|c|}
\hline \multirow{3}{*}{ Espécies } & \multicolumn{5}{|c|}{ Extrato aquoso } & \multicolumn{5}{|c|}{ Extrato hidroalcoólico } \\
\hline & \multicolumn{5}{|c|}{ Concentrações (\%) } & \multicolumn{5}{|c|}{ Concentrações (\%) } \\
\hline & $\mathbf{0}$ & 5 & 10 & 20 & $C V * \%$ & $\mathbf{0}$ & 5 & 10 & 20 & $C V * \%$ \\
\hline Fusarium oxysporum f. sp. phaseoli 1 & $9,00 a$ & $7,85 b$ & $6,80 \mathrm{c}$ & $4,10 \mathrm{~d}$ & 7,27 & $9,00 a$ & $6,90 \mathrm{~b}$ & $6,50 \mathrm{bc}$ & $5,95 \mathrm{c}$ & 5,44 \\
\hline F. oxysporum f. sp. phaseoli 2 & $9,00 a$ & $7,70 \mathrm{~b}$ & $7,10 \mathrm{~b}$ & $5,10 \mathrm{c}$ & 5,13 & $9,00 a$ & $8,15 b$ & $7,20 \mathrm{c}$ & $6,80 \mathrm{c}$ & 5,37 \\
\hline F. oxysporum f. sp. tracheiphilum & $9,00 a$ & $8,65 a$ & $7,25 b$ & $5,60 \mathrm{c}$ & 7,16 & $9,00 a$ & $7,40 b$ & $7,10 \mathrm{~b}$ & $5,80 \mathrm{c}$ & 6,11 \\
\hline
\end{tabular}

Na linha, mesma letra não difere estatisticamente entre si pelo teste Tukey $5 \%$ de probabilidade. CV*: Coeficiente de Variação 
(Tabela 2). David (2013) analisou a ação fungitóxica de extratos alcoólico e acético de mastruz em $F$. oxysporum f. sp. cubense e observou a inibição do crescimento micelial a partir da utilização do extrato a $5 \%$, porém o extrato alcoólico apresentou toxicidade, assim como observado no presente estudo, quando avaliado o efeito do extrato vegetal sobre $F$. oxysporum $\mathrm{f}$. sp. phaseoli e F. oxysporum f. sp. tracheiphilum. Freire et al. (2015) observaram que o extrato alcoólico de mastruz não foi eficaz para o controle de Lasiodiplodia sp. na concentração de $1 \%$, o que pode diferir principalmente devido à metodologia empregada e patógenos utilizados, além de outros fatores. Silva (2015) verificou a fungitoxidade de extrato alcoólico de mastruz, o qual conseguiu inibir $5,28 \%$ do crescimento micelial do F. oxysporum f. sp. passiflorae.

$\mathrm{O}$ extrato aquoso de agave não foi eficiente na redução da esporulação do isolado 1 de $F$. oxysporum f. sp. phaseoli e causou diminuição da esporulação em $F$. oxysporum $\mathrm{f}$. sp. phaseoli 2 nas concentrações de 5\% e 10\% (Tabela 3 ). $\mathrm{O}$ extrato hidroalcoólico de agave foi eficiente na redução da esporulação de F. oxysporum f. sp. tracheiphilum, sendo reduzida à medida em que a concentração do extrato aumentava (Tabela 4). Quanto ao isolado de F. oxysporum f. sp.phaseoli 1, apresentou redução apenas na concentração de $20 \%$, e o isolado F. oxysporum f. sp. phaseoli 2 apresentou redução na esporulação a partir da concentração de 5\% do extrato (Tabela 4).
O extrato aquoso de mastruz reduziu a esporulação de todos os isolados testados na concentração de 20\% (Tabela 5). No entanto, houve aumento na esporulação dos três isolados quando testadas as concentrações inferiores a $20 \%$, em que os isolados $F$. oxysporum f. sp. phaseoli 1 e $F$. oxysporum f. sp. tracheiphilum obtiveram máximos valores de esporulação em $5 \%$ da concentração do extrato. De modo semelhante F. oxysporum f. sp. phaseoli 2 aumentou a esporulação na concentração de 5\% e 10\% (Tabela 5).

Fontes (2012) testou extratos aquosos de alho (Allium sativum L.), alecrim (Rosmariunus officinalis L.), arruda (Ruta graveolens L.) e gengibre (Zingiber officinale Rosc.) nas concentrações (10\%, $20 \%$ e $30 \%$ ) sobre a esporulação de $F$. oxysporum f. sp. phaseoli, observando redução a partir da concentração de $10 \%$, com exceção do extrato de alecrim que reduziu significativamente a esporulação na concentração de 30\%. Pires (2015) avaliou o efeito de extratos aquosos de alecrim, alho, carqueja [Baccharis trimera (Less.) DC.] e vassoura-lageana (Baccharis uncinella DC) sobre a esporulação de Fusarium verticillioides e observou atividade tóxica apenas do extrato aquoso de alho, enquanto os extratos aquosos das outras espécies não inibiram a esporulação do fitopatógeno.

O extrato hidroalcoólico de mastruz aumentou a esporulação dos isolados de F. oxysporum $\mathrm{f}$ sp phaseoli 1 , no isolado 2 houve um aumento na esporulação. Para o isolado $F$. oxysporum f.

Tabela 3. Ação fungitóxica de extrato aquoso de agave sobre a esporulação de Fusarium oxysporum (1x 10 $\left.1{ }^{6} \operatorname{conidios} / \mathrm{ml}\right)$.

\begin{tabular}{lccccc}
\hline \multicolumn{1}{c}{ Fungos } & \multicolumn{3}{c}{ Concentração (\%) } \\
\cline { 2 - 5 } & 0 & 5 & 10 & 20 \\
\hline Fusarium oxysporum f. sp. phaseoli 1 & 15,49 aA & 13,98 aA & 14,66 aA & 13,67 aA \\
F oxysporum f. sp. phaseoli 2 & 12,44 bA & 07,02 bB & 06,95 bB & 10,60 bA \\
F. oxysporum f. sp. Tracheiphilum & 15,56 aA & 06,59 bB & $07,51 b B$ & 08,13 bB
\end{tabular}

$\mathrm{CV}^{*}$ : Coeficiente de Variação $=15,22 \%$; Mesma letra minúscula na coluna, não difere estatisticamente pelo teste; Mesma letra maiúscula na linha, não difere estatisticamente pelo teste; Tukey $(\mathrm{P} \leq 0.05)$.

Tabela 4. Ação fungitóxica de extrato hidroalcoólico de agave sobre a esporulação de Fusarium oxysporum (1x $10^{6}$ conidios $\left./ \mathrm{ml}\right)$.

\begin{tabular}{lccccc}
\hline \multicolumn{1}{c}{ Espécies } & \multicolumn{4}{c}{ Concentração (\%) } \\
\cline { 2 - 5 } & 0 & 5 & 10 & 20 \\
\hline Fusarium oxysporum f. sp. phaseoli 1 & $14,93 \mathrm{aA}$ & $15,42 \mathrm{aA}$ & $13,81 \mathrm{aAB}$ & $11,96 \mathrm{aB}$ \\
F. oxysporum f. sp. phaseoli 2 & $13,04 \mathrm{bA}$ & $06,17 \mathrm{bB}$ & $07,50 \mathrm{bB}$ & $06,96 \mathrm{bB}$ \\
F. oxysporum f. sp. tracheiphilum & $15,21 \mathrm{aA}$ & $06,70 \mathrm{bB}$ & $06,81 \mathrm{bB}$ & $03,38 \mathrm{cC}$ \\
\hline
\end{tabular}

$\mathrm{CV}^{*}$ : Coeficiente de Variação $=11,78 \%$; Mesma letra minúscula na coluna, não difere estatisticamente pelo teste; Mesma letra maiúscula na linha, não difere estatisticamente pelo teste; Tukey $(\mathrm{P} \leq 0.05)$.

Tabela 5. Ação fungitóxica de extrato aquoso de mastruz sobre a esporulação de Fusarium oxysporum.

\begin{tabular}{lccccc}
\hline \multicolumn{1}{c}{ Espécies } & \multicolumn{4}{c}{ Concentração (\%) } \\
\cline { 2 - 5 } & 0 & 5 & 10 & 20 \\
\hline Fusarium oxysporum f. sp. phaseoli 1 & $12,80 \mathrm{aB}$ & $38,86 \mathrm{aA}$ & $10,98 \mathrm{bB}$ & $\mathbf{2 . 0 6} \mathrm{bC}$ \\
F. oxysporum f. sp. phaseoli 2 & $13,58 \mathrm{aC}$ & $26,21 \mathrm{bB}$ & $39,25 \mathrm{aA}$ & $\mathbf{8 , 1 2} \mathrm{aD}$ \\
F. oxysporum f. sp. tracheiphilum & $13,46 \mathrm{aB}$ & $21,00 \mathrm{cA}$ & $5,08 \mathrm{cC}$ & $2,42 \mathrm{bC}$ \\
\hline
\end{tabular}

$\mathrm{CV}^{*}$ : Coeficiente de Variação $=14,65 \%$; Mesma letra minúscula na coluna, não difere estatisticamente pelo teste; Mesma letra maiúscula na linha, não difere estatisticamente pelo teste; Tukey $(\mathrm{P} \leq 0.05)$. 
Tabela 6. Ação fungitóxica de extrato hidroalcoólico de mastruz sobre a esporulação de Fusarium oxysporum.

\begin{tabular}{lcccc}
\hline \multicolumn{1}{c}{ Espécies } & \multicolumn{4}{c}{ Concentração (\%) } \\
\cline { 2 - 5 } & 0 & 5 & 10 & 20 \\
\hline Fusarium oxysporum f. sp. phaseoli 1 & 12,19 bC & 16,07 bB & $15,41 \mathrm{bB}$ & $21,87 \mathrm{bA}$ \\
F. oxysporum f. sp. phaseoli 2 & $15,83 \mathrm{aD}$ & $35,88 \mathrm{aA}$ & $31,33 \mathrm{aB}$ & $26,95 \mathrm{aC}$ \\
F. oxysporum f. sp. tracheiphilum & 14,38 abA & $12,08 \mathrm{cAB}$ & $10,31 \mathrm{cB}$ & $03,13 \mathrm{cC}$ \\
\hline
\end{tabular}

$\mathrm{CV}^{*}$ : Coeficiente de Variação $=9,90 \%$; Mesma letra minúscula na coluna, não difere estatisticamente pelo teste; Mesma letra maiúscula na linha, não difere estatisticamente pelo teste; Tukey $(\mathrm{P} \leq 0.05)$

sp. tracheiphilum, o extrato hidroalcoólico de mastruz reduziu a esporulação do fungo à medida que se aumentavam as concentrações (Tabela 6). David (2013) relata a atividade fungitóxica de extratos alcoólico e acético de mastruz sobre a esporulação de F. oxysporum f. sp. cubense, onde ambos os extratos foram eficientes a partir da concentração de 5\%; resultados semelhantes ao extrato hidroalcoólico de mastruz sobre Fusarium oxysporum f. sp. tracheiphilum no presente estudo, onde o mastruz também se mostrou eficaz na redução da produção de esporos.

\section{Conclusões}

O extrato aquoso de agave não reduz o crescimento micelial dos isolados de F. oxysporum f. sp. phaseoli 1 e F. oxysporum f. sp. tracheiphilum, mas aumentou o crescimento micelial do isolado de $F$. oxysporum f. sp. phaseoli 2. A esporulação de $F$. oxysporum f. sp. tracheiphilum é reduzida pelo extrato aquoso de agave, enquanto que o $F$. oxysporum f. sp. phaseoli 2 reduz nas concentrações $5 \%$ e $10 \%$.

O extrato hidroalcoólico de agave não apresenta eficácia na redução do crescimento micelial dos isolados testados em todas as concentrações avaliadas; entretanto, esse mesmo extrato consegue reduzir a esporulação dos isolados $F$. oxysporum f. sp. phaseoli 1 e 2, e F. oxysporum f. sp. tracheiphilum na concentração de $20 \%$ do extrato.

O extrato aquoso de mastruz aponta redução do crescimento micelial dos três isolados avaliados; a esporulação dos fungos pode ser afetada com o extrato aquoso de mastruz na concentração de $20 \%$ para todos os isolados testados. O extrato hidroalcoólico de mastruz reduz o crescimento micelial dos três isolados avaliados; o extrato hidroalcoólico de mastruz reduz a esporulação, apenas, em F. oxysporum f. sp. tracheiphilum.

\section{Agradecimentos}

À Fundação de Amparo à Ciência e Tecnologia do Estado de Pernambuco - Facepe, pela concessão de Bolsa de Iniciação Científica do primeiro autor, processo BIC-0951-5.01/19; ao Instituto Agronômico de Pernambuco - IPA, pelo suporte e pelo espaço utilizado para a realização dos experimentos; à Universidade Federal Rural de Pernambuco - UFRPE, pela minha formação, e a toda a equipe de trabalho.

\section{Referências}

Andresen, M. et al. Seed treatment with an aqueous extract of Agave sisalana improves seed health and seedling growth of sorghum.
European Journal of Plant Pathology, Dordrecht, v. 141, p. 119-132, 2015.

Bernardo, R. et al. Atividade antibacteriana de plantas medicinais. Summa Phytophathologica, Botucatu, v. 28, p. 1 -110, 2002.

Biazon, V. L. Crestamento bacteriano comum do feijoeiro: efeito da adubação nitrogenada e potássica e aspectos relacionados à doença. 2003. 171p. Dissertação (Mestrado em Ciências Agronômicas) Universidade Estadual Paulista, São Paulo, 2003.

Brito, M. M. P.; Muraoka, T.; Silva, E. C. Marcha de absorção do nitrogênio do solo, do fertilizante e da fixação simbiótica em feijãocaupi (Vigna unguiculata (1.) Walp.) e feijão-comum (Phaseolus vulgaris L.) determinada com uso de $15 \mathrm{~N}$. Revista Brasileira de Ciência do Solo, Viçosa, v. 33, p. 895-905, 2009.

Carnelossi, P. R. et al. Óleos essenciais no controle pós-colheita de Colletotrichum gloeosporioides em mamão. Revista Brasileira de Plantas Medicinais, Paulínia, v. 11, n. 4, p. 399-406, 2009.

Cavero, P. A. S. et al. Biological control of banana black Sigatoka disease with Trichoderma. Ciência Rural, Santa Maria, v. 45, p. 951-957, 2015.

CELOTO, M. I. B. et al. Atividade antifúngica de extratos de plantas a Colletotrichum gloeosporioides. Acta Scientiarum: Agronomy, Maringá, v. 30, p. 1-5, 2008.

Chand, N.; Tiwary, R. K.; Rohatgi, P. K. Bibliography resource structure properties of natural cellulosic fibres-an annotated bibliography. Journal of Materials Science, USA, v. 23, n. 2, p. 381-387, 1988. http://dx.doi.org/10.1007/BF01174659.

COMPAINHA NACIONAL DE ABASTECIMENTO - CONAB. Perspectivas para a Agropecuária. Brasília: CONAB, 2014. v. 1 - Safra 2013/2014 - Edição Grãos. Disponivel em: <https:// www.conab.gov.br/perspectivas-para-a-agropecuaria $>$. Acesso em: 7 out. 2021.

David, N. A. Avaliação do efeito antifúngico de extratos de plantas no controle do Fusarium oxysporium f. sp. cubense in vitro e em rizomas de Heliconia sp. 2013. 60 f. Monografia (Graduação em Agronomia)-Curso de Agronomia, Universidade Federal do Amazonas, Humaitá, 2013.

Domingues, R. J. et al. Ação in vitro de extratos vegetais sobre Colletrotichum acutatum, Alternaria solani e Sclerotium rolfsii. Arquivo do Instituto de Biologia, São Paulo, v. 76, p. 643-649, 2009.

Edginton, L. V.; Knew, K. L.; Barron, G. L. Fungitoxic spectrum of benzimidazole compounds. Phytopathology, Mineaussota, v. 62, p. 42-44, 1971.

FOOD AND AGRICULTURE ORGANIZATION OF THE UNITED NATIONS - FAOSTAT. Colheitas (Crops). 2018. Disponível em: $<$ http://www.fao.org/faostat/en/\#data/QC $>$. Acesso em: 8 out. 2021.

Ferreira, D. F. SISVAR: A computer analysis system to fixed effects split plot type designs. Revista Brasileira De Biometria, Lavras, v. 37, n. 4, p. 529-535, 2019. https://doi.org/10.28951/rbb.v37i4.450. 
Fontes, A. C. L. Variabilidade genética e avaliação da inibição dos extratos de plantas medicinais sobre isolados de Fusarium oxysporum f. sp. phaseoli. 2012 71p. (Dissertação de Mestrado em Biologia de Fungos)-Universidade Federal de Pernambuco, Recife, 2012.

Freire, A. N. R. et al. Efeito de extratos de plantas medicinais sobre Lasiodiplodia sp. In: $19^{\circ}$ Seminário de Iniciação Científica, 19., Seminário de Pós-graduação da Embrapa Amazônia Oriental, 3., Pará. Anais... Belém: Embrapa Amazônia Oriental, 2015.

Frota, K. M. G. et al. Cholesterol-lowering properties of whole cowpea seed and its protein isolate in hamsters. Journal of Food Science, Chicago, v. 73, p. H235-H240, 2008.

Ghini, R., KIMATI, H. Resistência de fungos a fungicidas. Jaguariúna: Embrapa Meio Ambiente, 2000.

Lopes, R. S. et al. Efficacy of Libidibia ferrea var. ferrea and Agave sisalana extracts against Dactylopius opuntiae (Hemiptera: Coccoidea). The Journal of Agricultural Science, Canada, v. 10, n. 4, p. 255-267, 2018. http://dx.doi.org/10.5539/jas.v10n4p255.

Marins, A. K. et al. Prospecção fitoquímica das Partes Aéreas da erva-de-santa-maria (Chenopodium ambrosioides L.). In: Encontro Latino Americano de Iniciação Científica, 15., Encontro Latino Americano de Pós-Graduação, 6., Encontro Latino Americano de Iniciação Científica Jr., 5.. Anais... São José dos Campos: Univap, 2011.

Milanesi, P. M. et al. Ação fungitóxica de extratos vegetais sobre o crescimento micelial de Colletotrichum gloeosporioides. Revista da FZVA, Uruguaiana, v. 16, n. 1, p. 1-13, 2009.

Morais, M. S. et al. Eficiência dos extratos de alho e agave no controle de Fusarium oxysporum S. Revista Brasileira de Agroecologia, Dois Vizinhos, v. 5, n. 2, p. 89-98, 2010.

Pires, A. F. Atividade antifúngica de plantas medicinais sobre o desenvolvimento de Fusarium verticillioides em sementes de milho crioulo. 2015. 40 f Trabalho de Conclusão (Graduação em Agronomia)-Universidade Federal de Santa Catarina, Florianópolis, 2015.

Romeiro, R. S. Indução de resistência em plantas a patógenos. In: PASCHOLATI, S. F. et al. Interação planta-patógeno: fisiologia, bioquímica e biologia molecular. Piracicaba: FEALQ, 2008. p. 411-429.

Sartorato, A. Desafios no controle de doenças na cultura do feijoeiro na região Centro-Oeste. In: Ito, M. F.; Stein, C. P. (Eds.). VI Seminário sobre pragas, doenças e plantas daninha do feijoeiro. Campinas (SP): Instituto Agronômico de Campinas (IAC). p.15-17,2007.

Schwan-Estrada, K. R. F.; Stangarlin, J. R. Extratos e óleos essenciais de plantas medicinais na indução de resistência. In: Cavalcanti, L.S. et al. (Eds.). Indução de resistência em plantas a patógenos e insetos. Piracicaba: FEALQ, 2005. p. 125-132.

Silva, C. T. B. Estudo dos sistemas produtivos da Cooperativa Agropecuária dos Produtores Familiares Irituienses e o potencial de extratos de plantas medicinais no manejo de pragas e doenças do maracujazeiro. 2015, 107 f. Dissertação (Mestrado em Agriculturas Familiares e Desenvolvimento Sustentável)-Universidade Federal do Pará, Pará, 2015.

Silva, D. M. M. H.; Bastos, C. N. Atividade antifúngica de óleos essenciais de espécies de Piper sobre Crinipellis perniciosa, Phytophthora palmivora e Phytophthora capsici. Fitopatologia Brasileira, Brasília, v. 32, n. 2, p. 143-145, 2007. http://dx.doi. org/10.1590/S0100-41582007000200008.

Valadão, L. T.; Klar, A. E. Evapotranspiração do feijoeiro (Phaseolus vulgaris L.) em dois níveis do lençol freático. In: CONGRESSO NACIONAL DE IRRIGAÇÃO E DRENAGEM, 1996, Campinas-SP. Anais... Abid/Cati, 1996.

Venturoso, L. R. et al. Atividade antifúngica de extratos vegetais sobre o desenvolvimento de fitopatógenos. Summa Phytopathologica, Jaguariúna, v. 37, n. 1, p. 18-23, 2011. http://dx.doi.org/10.1590/ S0100-54052011000100003.

Wendland JUNIOR, A.; LOBO JUNIOR, M.; Faria, J. C. Manual de identificação das principais doenças do feijoeiro-comum. Brasília, DF: Embrapa Arroz e Feijão, 2018. 